J. Korean Math. Soc. 52 (2015), No. 1, pp. 159-176

http://dx.doi.org/10.4134/JKMS.2015.52.1.159

\title{
TOTAL IDENTITY-SUMMAND GRAPH OF A COMMUTATIVE SEMIRING WITH RESPECT TO A CO-IDEAL
}

\author{
Shahabaddin Ebrahimi Atani, Saboura Dolati Pish Hesari, \\ AND MEHDI KHORAMDEL

\begin{abstract}
Let $R$ be a semiring, $I$ a strong co-ideal of $R$ and $S(I)$ the set of all elements of $R$ which are not prime to $I$. In this paper we investigate some interesting properties of $S(I)$ and introduce the total identity-summand graph of a semiring $R$ with respect to a co-ideal $I$. It is the graph with all elements of $R$ as vertices and for distinct $x, y \in R$, the vertices $x$ and $y$ are adjacent if and only if $x y \in S(I)$.
\end{abstract}

\section{Introduction}

The concept of a zero-divisor graph of a commutative ring was introduced by Beck in [5]. In his work all elements of the ring were vertices of the graph. In [3], Anderson and Livingston introduced and studied the zero-divisor graph whose vertices are the non-zero zero-divisors of a ring. In [2], the authors defined the total graph of a ring $R$ to be the (undirected) graph $T(\Gamma(R))$ with all elements of $R$ as vertices, and two distinct vertices $x$ and $y$ are adjacent if and only if $x+y \in Z(R)$, where $Z(R)$ is the zero divisors of $R$. Also they studied the subgraph $T_{0}(\Gamma(R))$ of $T(\Gamma(R))$ with vertices $R \backslash\{0\}$.

Recently, the study of graphs of rings was extended to include semirings as in $[7,8,9]$. Semirings have proven to be useful in theoretical computer science, in particular for studying automata and formal languages. Moreover, co-ideals of semirings play an important role in the structure theory and useful for many purposes. They have properties that are more suited than the properties of ideals, to the study of the graphs of semirings, such as Proposition 2.1(ii) and Theorem 3.7. In $[11,12]$, the authors introduced the identity-summand graph and identity-summand graph with respect to co-ideal $I$ of a semiring $R$. The identity-summand graph with respect to co-ideal $I$ denoted by $\Gamma_{I}(R)$ is a graph

Received May 9, 2014; Revised July 12, 2014.

2010 Mathematics Subject Classification. Primary 16Y60, 05 C62.

Key words and phrases. strong co-ideal, total identity-summand graph, identity-summand graph based a co-ideal, total identity-summand graph based a co-ideal. 
with vertices as elements $S_{I}(R)=\{x \in R \backslash I: x+y \in I$ for some $y \in R \backslash I\}$, where two distinct vertices $x$ and $y$ are adjacent if and only if $x+y \in I$ [12].

We say that $r \in R$ is an identity-summand element of $R$, if there exists $1 \neq a \in R$ such that $r+a=1$. The notation $S(R)$ is used to refer to the set of elements of $R$ that are identity-summand (we use $S^{*}(R)$ to denote the set of non-identity identity-summands of $R$ ). A semiring $R$ is called an $I$-semiring if $r+1=1$ for all $r \in R$ [10]. In [13], the authors introduced the total graph of a commutative semiring with respect to identity-summand elements. Let $R$ be an $I$-semiring. The total graph of $R$, denoted by $T(\Gamma(R))$, is the graph with all elements of $R$ as vertices, and for distinct $x, y \in R$, the vertices $x$ and $y$ are adjacent if and only if $x y \in S(R) . S(\Gamma(R))$ (resp. $S^{*}(\Gamma(R))$ ) denotes the subgraph of $T(\Gamma(R))$ with vertex set $S(R)$ (resp. $S^{*}(R)$ ). In this paper the authors extend the results obtained in [13] for a subtractive co-ideal $I$ of $R$ and it is shown that these results do not hold for a non-subtractive co-ideal.

Let $I$ be a co-ideal of $R$, we say that $a \in R$ is prime to $I$, if $r+a \in I$ (where $r \in R$ ) implies that $r \in I$ and we define the set of elements of $R$ which are not prime to $I$ by $S(I)$. The set $S(I)$ is not necessarily a co-ideal of $R$. In this paper we prove some important properties of $S(I)$ which are so useful in solving the problems of the total graph with respect to a co-ideal of $R$.

In Section 3, we show that if $S(I)$ is finite, then $S(I)$ is not a co-ideal of $R$. In Lemma 3.1(ii), we show that $S(I)$ is a union of subtractive prime co-ideals of $R$ and in Theorem 3.7, we show that $S(I)$ is a union of minimal prime co-ideals of $I$. Also, it is shown that if $\min (I)$ is finite, then the set of subtractive prime co-ideals which construct $S(I)$ in Lemma 3.1 is equal to the set of minimal prime co-ideals of $R$. But if $\min (I)$ is infinite, this equality is not true, as the Example 3.12 shows.

In Section 4, we introduce and study the total graph of $R$ with respect to co-ideal $I$. At the beginning of the section, it is shown that $\bar{S}\left(\Gamma_{I}(R)\right)$ (the subgraph of $T\left(\Gamma_{I}(R)\right.$ ) with vertex set $R \backslash S(I)$ ) is totally disconnected, which implies $T\left(\Gamma_{I}(R)\right)$ is always disconnected. So we investigate only the subgraph $S\left(\Gamma_{I}(R)\right)$ of $T\left(\Gamma_{I}(R)\right)$ (the induced subgraph of $T\left(\Gamma_{I}(R)\right)$ with vertex set $S(I))$. We show that $S\left(\Gamma_{I}(R)\right)$ is connected, $\operatorname{diam}\left(S\left(\Gamma_{I}(R)\right)\right) \in\{1,2\}$ and $\operatorname{gr}\left(S\left(\Gamma_{I}(R)\right)\right) \in\{3, \infty\}$. Moreover, we investigate $\kappa\left(S\left(\Gamma_{I}(R)\right)\right)$ and the cut points of $S\left(\Gamma_{I}(R)\right)$ and we consider when $S\left(\Gamma_{I}(R)\right)$ has a cut point. At the end of the section, it is proved that if $S\left(\Gamma_{I}(R)\right) \cong S\left(\Gamma_{J}(T)\right)$, then $\Gamma_{I}(R) \cong \Gamma_{J}(T)$, where $R$ and $S$ are semirings and $I, J$ are co-ideals of $R$ and $S$, respectively.

In Section 5, we describe the relation between $S\left(\Gamma_{I}(R)\right)$ and $S(\Gamma(R / I))$ for a $Q$-strong co-ideal of $R$. It is shown that $\operatorname{diam}\left(S\left(\Gamma_{I}(R)\right)\right)=1$ if and only if $\operatorname{diam}(S(\Gamma(R / I)))=1$ and $\operatorname{diam}\left(S\left(\Gamma_{I}(R)\right)\right)=2$ if and only if $\operatorname{diam}(S(\Gamma(R / I)))$ $=2$. 


\section{Preliminaries}

In order to make this paper easier to follow, we recall various notions which will be used in the sequel. For a graph $\Gamma$ by $E(\Gamma)$ and $V(\Gamma)$ we denote the set of all edges and vertices, respectively. A graph $G$ is called connected if for any vertices $x$ and $y$ of $G$ there is a path between $x$ and $y$. Otherwise, $G$ is called disconnected. The distance between two distinct vertices $a$ and $b$, denoted by $d(a, b)$, is the length of the shortest path connecting them (if such a path does not exist, then $d(a, b)=\infty$, also $d(a, a)=0)$. The diameter of graph $\Gamma$, denoted by $\operatorname{diam}(\Gamma)$, is equal to $\sup \{d(a, b): a, b \in V(\Gamma)\}$. A graph is complete if it is connected with diameter less than or equal to one. A clique of a graph is its complete subgraph and the number of vertices in the largest clique of graph $G$, denoted by $w(G)$, is called the clique number of $G$.

A commutative semiring $R$ is defined as an algebraic system $(R,+, \cdot)$ such that $(R,+)$ and $(R, \cdot)$ are commutative semigroups, connected by $a(b+c)=$ $a b+a c$ for all $a, b, c \in R$, and there exist $0,1 \in R$ such that $r+0=r$ and $r 0=$ $0 r=0$ and $r 1=1 r=r$ for each $r \in R$. In this paper all semirings considered will be assumed to be commutative semirings with a non-zero identity.

Definition. Let $R$ be a semiring.

(1) A non-empty subset $I$ of $R$ is called a co-ideal, if it is closed under multiplication and satisfies the condition $r+a \in I$ for all $a \in I$ and $r \in R$ (so $0 \in I$ if and only if $I=R$ ). A co-ideal $I$ of $R$ is called strong co-ideal provided that $1 \in I[10,15,17]$.

(2) If $I$ is a co-ideal of $R$, then the $\operatorname{co-rad}(I)$ of $I$, is the set of all $x \in R$ for which $n x \in I$ for some positive integer $n$. This is a co-ideal of $R$ contains $I$ [10].

(3) A co-ideal $I$ of $R$ is called subtractive if $x, x y \in I$, then $y \in I$ (so every subtractive co-ideal is a strong co-ideal) [10].

(4) A proper co-ideal $P$ of $R$ is called prime if $x+y \in P$, then $x \in P$ or $y \in P$. The set of all prime (resp. minimal prime) co-ideals of $R$ is denoted by $\operatorname{co-Spec}(R)(\operatorname{resp} \cdot \min (R))[10]$.

(5) A semiring $R$ is called co-semidomain, if $a+b=1(a, b \in R)$, then either $a=1$ or $b=1[10]$.

(6) We say that a subset $T \subseteq R$ is additively closed if $0 \in T$ and $a+b \in T$ for all $a, b \in T$.

(7) An ideal $I$ of $R$ is called $k$-ideal if $x, x+y \in I$, then $y \in I$ for all $x, y \in R$ $[14]$.

A strong co-ideal $I$ of a semiring $R$ is called a partitioning strong co-ideal (= $Q$-strong co-ideal) if there exists a subset $Q$ of $R$ such that $R=\cup\{q I: q \in Q\}$, where $q I=\{q t: t \in I\}$ and if $q_{1}, q_{2} \in Q$, then $\left(q_{1} I\right) \cap\left(q_{2} I\right) \neq \emptyset$ if and only if $q_{1}=q_{2}$ [10]. Let $I$ be a $Q$-strong co-ideal of a semiring $R$ and let $R / I=\{q I: q \in Q\}$. Then $R / I$ forms a semiring under the binary operations $\oplus$ and $\odot$ defined as follows: $\left(q_{1} I\right) \oplus\left(q_{2} I\right)=q_{3} I$, where $q_{3}$ is the unique element 
in $Q$ such that $\left(q_{1} I+q_{2} I\right) \subseteq q_{3} I$, and $\left(q_{1} I\right) \odot\left(q_{2} I\right)=q_{3} I$, where $q_{3}$ is the unique element in $Q$ such that $\left(q_{1} q_{2}\right) I \subseteq q_{3} I$ [10]. If $q_{e}$ is the unique element in $Q$ such that $1 \in q_{e} I$, then $q_{e} I=I$ is the identity of $R / I$. Note that every $Q$-strong co-ideal is subtractive [10]. Throughout this paper we shall assume unless otherwise stated, that $q_{e} I$ is the identity element of $R / I$.

Proposition 2.1. (i) [11, Proposition 2.5] Let $R$ be a commutative I-semiring. Then the following statements hold:

(1) If $a+a=1$ for some $a \in R$, then $a=1$;

(2) If $J$ is a co-ideal, then $J$ is a strong co-ideal of $R$. Moreover, if $x y \in J$, then $x, y \in J$ for every $x, y \in R$. In particular, $J$ is subtractive;

(3) The set $(1: x)=\{r \in R: r+x=1\}$ is a strong co-ideal of $R$ for every $x \in S(R)$.

(ii) $[12$, Proposition 2.1] Let $I$ be a subtractive co-ideal of a semiring $R$. Then the following hold:

(1) If $x y \in I$, then $x, y \in I$ for all $x, y \in R$;

(2) $I=\operatorname{co}-\operatorname{rad}(I)$;

(3) $(I: a)=\{r \in R: r+a \in I\}$ is a subtractive co-ideal of $R$ for all $a \in R$;

(4) If $I$ is a $Q$-strong co-ideal of $R$ and $q_{e} I$ is the identity element in $R / I$, then $q_{e} I \oplus q I=q_{e} I$ and $q I \oplus q I=q I$ for all $q I \in R / I$.

(iii) [12, Theorem 4.6] Let $I$ be a subtractive co-ideal of a semiring $R$.

(1) If $\left\{P_{\alpha}\right\}_{\alpha \in \Lambda}$ is the set of all prime strong co-ideals of $R$ containing $I$, then $I=\cap_{\alpha \in \Lambda} P_{\alpha}$.

(2) If $P_{1}, \ldots, P_{n}$ are the only distinct minimal prime strong co-ideals of $R$ containing $I$, then $\cap_{i=1}^{n} P_{i}=I$ and $I \neq \cap_{1 \leq i \leq n, i \neq j} P_{i}$ for each $1 \leq j \leq n$.

Proposition 2.2. (i) [12, Theorem 2.8] If $I$ is a subtractive co-ideal of $R$ with $\left|S\left(\Gamma_{I}(R)\right)\right| \geq 3$, then $\Gamma_{I}(R)$ is not a complete graph. In particular, $\operatorname{diam}\left(\Gamma_{I}(R)\right)=2$ or 3 .

(ii) [13, Theorem 4.4] Let $R$ be an I-semiring which is not co-semidomain. Then $S^{*}(\Gamma(R))$ is connected if and only if $|\min (R)| \neq 2$. Moreover if $S^{*}(\Gamma(R))$ is connected, then $\operatorname{diam}\left(S^{*}(\Gamma(R))\right) \in\{1,2\}$.

(iii) [12, Theorem 4.7] If $I$ is a subtractive co-ideal of $R$ which is not prime, then $w\left(\Gamma_{I}(R)\right)=|\min (I)|$.

\section{Some properties of $S(I)$}

In this section we introduce $S(I)$, the set of elements of $R$ which are not prime to $I$. We give an interesting lemma which will be useful in next sections. Also, we investigate one of the most important properties of $S(I)$, with respect to minimal prime co-ideals of $I$. We begin with the following definition.

Definition. Let $R$ be a semiring and $I$ be a strong co-ideal of $R$. We define $S(I)$ by $S(I)=\{r \in R: \exists x \in R \backslash I$ such that $r+x \in I\}$ and $\bar{S}=R \backslash S(I)$.

Compare the following lemma with [13, Lemma 3.6 and Lemma 2.4]. 
Lemma 3.1. Let $R$ be a semiring and $I$ be a subtractive co-ideal of $R$. The following statements hold:

(i) If $I \neq S(I)$, then $|S(I)| \neq 1,2$. Moreover if $S(I)$ is finite, then $S(I)$ is not a co-ideal of $R$.

(ii) $S(I)$ is a union of subtractive prime co-ideals of $R$ containing $I$. Moreover, if $x y \in S(I)$, then $x, y \in S(I)$ for each $x, y \in R$.

(iii) If $S(I)$ is a co-ideal of $R$, then $S(I)$ is a prime co-ideal of $R$.

(iv) $\bar{S}(I)$ is an ideal of $R$.

(v) $S(I)$ is a co-ideal of $R$ if and only if $\bar{S}(I)$ is a prime ideal of $R$.

(vi) $Q_{i}=R \backslash P_{i}$ is a prime $k$-ideal of $R$ for each $P_{i} \in \min (I)$.

(vii) $\bar{S}(I)=\cap_{i \in \Lambda} Q_{i}$.

Proof. (ii) Set

$\sum=\{J: J$ is subtractive and each element of $J$ is not prime to $I$ and $I \subseteq J\}$.

Since $I \in \sum, \sum \neq \emptyset$. By Zorn's lemma, $\sum$ has a maximal element. Let $P$ be a maximal element of $\sum$. We show that $P$ is a prime co-ideal of $R$. Let $x+y \in P$ and $x, y \notin P$. Since $P \subset(P: x)$ and $P$ is maximal in $\sum,(P: x) \notin \sum$. So there exists $z \in(P: x)$ such that $z$ is prime to $I$. We show that $(P: z) \in \sum$. Let $w \in(P: z) \backslash I$. Since $z$ is prime to $I, w+z \notin I$. Because $w+z \in P$, $w+z+u \in I$ for some $u \notin I$, which implies $w+u \in I$ because $z$ is prime to $I$. Thus $w$ is not prime to $I$ because $u \notin I$. Hence $(P: z) \in \sum$, a contradiction, because $P \subset(P: z)$ and $P$ is maximal in $\sum$. Thus $P$ is a prime co-ideal of $R$. Since $S(I)$ is a union of maximal elements of $\sum, S(I)$ is a union of prime coideals of $R$. Now, let $x y \in S(I)$. So $x y \in P_{i}$ for some $P_{i} \in \sum$. By Proposition 2.1(ii), $x, y \in P_{i} \subseteq S(I)$.

Other parts are similar to the proof of [13, Lemma 3.6 and Lemma 2.4].

In the next example we show that the condition " $I$ is subtractive" is not superfluous in Lemma 3.1(ii).

Example 3.2. Assume that $R=\{0,1,2,3,4,5\}$. Define

$$
a+b= \begin{cases}5 & \text { if } a \neq 0, b \neq 0, a \neq b \\ a & \text { if } a=b \\ b & \text { if } a=0 \\ a & \text { if } b=0\end{cases}
$$

and

$$
a * b= \begin{cases}0 & \text { if } a=0 \text { or } b=0, \\ 3 & \text { if } a=b=2, \\ b & \text { if } a=1, \\ a & \text { if } b=1, \\ 5 & \text { otherwise. }\end{cases}
$$

Then $(R,+, *)$ is easily checked to be a commutative semiring. An inspection will show that $I=\{1,4,5\}$ is a co-ideal of $R$ which is not subtractive (because 
$2 * 4=5 \in I$ and $2 \notin I)$. It can be easily seen that $S(I)=\{1,2,3,4,5\}$. As we see $S(I)$ is finite but $S(I)$ is a co-ideal of $R$.

We next give several lemmas in order to gain Theorem 3.7.

Lemma 3.3. Let $R$ be a semiring and $I$ be a subtractive co-ideal of $R$. If $T$ is an additively closed subset of $R$ such that $T \cap I=\emptyset$, then $\sum=\{S: T \subseteq$ $S, S$ is an additively closed subset of $R$ and $S \cap I=\emptyset\}$ has a maximal element.

Proof. It is straightforward by Zorn's lemma.

Lemma 3.4. Let $R$ be a semiring and $I$ be a subtractive co-ideal of $R$. If $T$ is an additively closed subset of $R$ such that $I \cap T=\emptyset$, then there exists a subtractive prime co-ideal $Q$ containing $I$ which is maximal with respect to the property of not meeting $T$.

Proof. Let $\sum=\{J: I \subseteq J, J$ is a subtractive co-ideal of $R$ and $J \cap T=\emptyset\}$. Since $I \in \sum, \sum \neq \emptyset$. By Zorn's lemma $\sum$ has a maximal element $Q$. We show that $Q$ is a prime co-ideal of $R$. Let $a+b \in Q$ and $a \notin Q$. So $Q \subset(Q: b)$. Since $Q$ is maximal in $\sum$, there exists $s \in T$ such that $s \in(Q: b) \cap T$. We show that $Q=(Q: a s)$. If not, then $Q \subset(Q: a s)$ implies that $(Q: a s) \cap T \neq \emptyset$. Let $r \in(Q: a s) \cap T$. Then as $\in(Q: r)$. By Proposition 2.1(ii), $s \in(Q: r)$. So $s+r \in Q \cap T$, a contradiction. Thus $Q=(Q: a s)$. We claim that $(Q: a s)=$ $(Q: a) \cap(Q: s)$. Since $Q=(Q: a s),(Q: a s) \subseteq(Q: a) \cap(Q: s)$. Now, let $r \in(Q: a) \cap(Q: s)$. Thus $a, s \in(Q: r)$. Hence $a s \in(Q: r)$ by Proposition 2.1(ii), which gives $r \in(Q: a s)$, as needed. Thus $(Q: a s)=(Q: a) \cap(Q: s)$. As $b \in(Q: a) \cap(Q: s), b \in Q$. Therefore $Q$ is a prime co-ideal of $R$.

Let $S$ be a subset of $R$. We denote the set of elements of $R \backslash S$ by $S^{c}$.

Lemma 3.5. Let $R$ be a semiring, $I$ be a subtractive co-ideal of $R$ and $P$ be a prime co-ideal of $R$. Then $P \in \min (I)$ if and only if $P^{c}$ is an additively closed subset of $R$ which is maximal with respect to the property of not meeting $I$. Moreover, every minimal prime co-ideal of $I$ is subtractive.

Proof. Let $P$ be a prime co-ideal of $R$ which $P^{c}$ is maximal with respect to the property of not meeting $I$. We show that $P \in \min (I)$. Let $Q \subseteq P$, where $Q$ is a prime co-ideal of $R$ containing $I$. The definition of a prime co-ideal implies that $Q^{c}$ is an additively closed subset of $R$ and $P^{c} \subseteq Q^{c}$. Since $P^{c}$ is maximal with respect to the property of not meeting $I, I \cap Q^{c} \neq \emptyset$. Let $x \in I \cap Q^{c}$, then $x \in I$ and $x \notin Q$, a contradiction. So $P \in \min (I)$.

Conversely, let $P \in \min (I)$, so $I \cap P^{c}=\emptyset$. We claim $P^{c}$ is maximal with respect to the property of not meeting $I$ : by Lemma 3.3, there exists a maximal additively closed subset $M$ of $R$ with respect to the property of not meeting $I$ such that $P^{c} \subseteq M$. By the proof of Lemma 3.4, there exists a subtractive prime co-ideal $Q$ containing $I$, which is maximal with respect to the property of not meeting $M$. Hence $Q \cap M=\emptyset$ and $Q \subseteq M^{c}$, so $Q \subseteq M^{c} \subseteq P$. Since $Q$ is prime and $P \in \min (I), Q=M^{c}=P$. Hence $P^{c}=M$ is maximal with 
respect to the property of not meeting $I$. For the moreover statement, since $Q$ is subtractive, $P$ is subtractive too.

Proposition 3.6. Let $R$ be a semiring, $I$ be a subtractive co-ideal of $R$ and $P$ a prime co-ideal of $R$. Then $P \in \min (I)$ if and only if for each $x \in P$ there exists $y \notin P$ and a positive integer $i$ such that $y+i x \in I$.

Proof. Assume the condition holds, we show $P \in \min (I)$. Let $Q$ be a prime co-ideal of $R$ containing $I$, which $Q \subseteq P$. Choose $x \in P \backslash Q$. By assumption, there exists $y \notin P$ and a positive integer $i$ such that $y+i x \in I$. Since $I \subseteq Q$ and $Q$ is prime, $i x \in Q$, which implies $x \in Q$, a contradiction. Thus $P \in \min (I)$.

Conversely, let $P \in \min (I)$. Let $x \in P$ and $T=\left\{y+i x: y \in P^{c}, i \in \mathbb{N} \cup\{0\}\right\}$ (Note that $0 x=0$ ). Then $T$ is an additively closed subset of $R$ which properly contains $P^{c}$. By Lemma 3.5, $P^{c}$ is maximal with respect to property not meeting $I$. Thus $I \cap T \neq \emptyset$. Hence there exists a positive integer $i$ and $y \notin P$ such that $y+i x \in I$.

Now we are in a position to prove our main theorem in this section.

Theorem 3.7. Let $R$ be a semiring and $I$ be a subtractive co-ideal of $R$. Then $S(I)=\cup_{P_{\alpha} \in \min (I)} P_{\alpha}$.

Proof. Let $P_{\alpha}$ be a minimal prime co-ideal of $I$ and $x \in P_{\alpha} \backslash I$. By Proposition 3.6, there exists $y \notin P_{\alpha}$ such that $y+i x \in I$ for some integer $i \neq 0$. Since $I$ is subtractive and $i x=(1+1+\cdots+1) x, x \in(I: y)$ by Proposition 2.1(ii). So $x+y \in I$, which gives $x \in S(I)$. Thus $\cup_{P_{\alpha} \in \min (I)} P_{\alpha} \subseteq S(I)$. Now, let $x \in S(I) \backslash I$, so there exists $y \in R \backslash I$ such that $x+y \in I$. Since $y \notin I$, there exists $P_{\alpha} \in \min (I)$ such that $y \notin P_{\alpha}$, because $\cap_{P_{\alpha} \in \min (I)} P_{\alpha}=I$, by Proposition 2.1(iii). Since $x+y \in I \subseteq P_{\alpha}$ and $y \notin P_{\alpha}, x \in P_{\alpha}$. Thus $S(I)=\cup_{P_{\alpha} \in \min (I)} P_{\alpha}$.

In the next example we show that the condition " $I$ is subtractive" can not be omitted in Theorem 3.7.

Example 3.8. Let $R$ be the semiring of Example 3.2 and $I=\{1,4,5\}$. So $P=\{1,3,4,5\}$ is the only minimal prime co-ideal of $I$. It can be easily seen that $S(I)=\{1,2,3,4,5\}$. As we see, $I \neq \cap_{P \in \min (I)} P$ and $S(I) \neq \cup_{P \in \min (I)} P$.

In Lemma 3.1 and Theorem 3.7, we see two set of prime co-ideals for $S(I)$. In the following we answer to this question that are two set of prime co-ideals for $S(I)$ equal?

The following lemma is useful in the proof of next corollary.

Lemma 3.9. Let $P_{1}, P_{2}, \ldots, P_{n}$ be subtractive prime co-ideals of a semiring $R$. If $I$ is a strong co-ideal of $R$ such that $I \subseteq \cup_{i=1}^{n} P_{i}$, then $I \subseteq P_{r}$ for some $1 \leq r \leq n$.

Remark 3.10. Let $P$ and $I$ be strong co-ideals of a semiring $R$ with $P$ prime and $I \subseteq P$. Then the non-empty set $\Delta=\{Q \in S \sec (R): I \subseteq Q \subseteq P\}$ has 
a minimal element $P_{1}$ with respect to inclusion (by partially ordering $\Delta$ by reverse inclusion and using Zorn's Lemma), so $P_{1}$ is an element of $\min (I)$, the set of minimal prime strong co-ideals of $R$ containing $I$. Thus if $P$ is a prime strong co-ideal of the commutative semiring $R$ and $P$ contains the strong coideal $I$ of $R$, then there exists a minimal prime strong co-ideal $Q$ of $R$ with $I \subseteq Q \subseteq P$.

Corollary 3.11. Let $R$ be a semiring and $I$ be a subtractive co-ideal of $R$. If $\min (I)$ is finite, then two sets of prime co-ideals which are defined in Theorem 3.7 and Lemma 3.1 are equal. So $S(I)=\cup P_{i}$, where $P_{i}^{\prime}$ s are subtractive minimal prime co-ideals of $R$ and are maximal in

$$
\sum=\{J \text { : each element of } J \text { is not prime to } I \text { and } I \subseteq J\} .
$$

Proof. Let $P_{i} \in \min (I)$, we show that $P_{i}$ is maximal in $\sum$. If $P_{i}$ is not maximal in $\sum$, there exists a maximal element $Q$ in $\sum$ such that $P_{i} \subset Q$. Because $Q \subseteq S(I)$ and $S(I)=\cup_{i=1}^{n} P_{i}$ by Theorem 3.7, $Q \subseteq P_{j}$ for some minimal prime co-ideal $P_{j}$ of $I$ by Lemma 3.9. So $P_{i}=Q=P_{j}$, a contradiction. So each minimal prime co-ideal of $I$ is maximal in $\sum$. Conversely, let $Q$ be maximal in $\sum$. If $Q \notin \min (I)$, there exists $P_{i} \in \min (I)$ such that $P_{i} \subseteq Q$ by Remark 3.10. But we showed that each minimal prime co-ideal of $I$ is maximal in $\sum$, hence $P_{i}=Q$.

The following example shows that the condition $\min (I)$ is finite can not be omitted in Corollary 3.11.

Example 3.12. Let $R=\left(\mathbb{Z}^{+}, \operatorname{gcd}, \mathrm{lcm}\right)($ take $\operatorname{gcd}(0,0)=0$ and $\operatorname{lcm}(0,0)=0)$. It is clear that $I=\{1\}$ and $S(I)=\mathbb{Z}^{+} \backslash\{0\}$ are co-ideals of $R$. We show that $\min (I)$ is infinite. Suppose, on the contrary, $\min (I)$ is finite. Hence $S(I) \subseteq P_{i}$ for some $P_{i} \in \min (I)$ by Lemma 3.9, which implies $S(I)=P_{i}$. Thus $P_{i}$ is the only minimal prime co-ideal of $R$ by Theorem 3.7. So $S(I)=P_{i}=I$ by Proposition 2.1(iii), a contradiction. Therefore $\min (I)$ is infinite. It is clear that each co-ideal of $R$ is contained in the maximal co-ideal $S(I)$ of $R$. Thus $S(I)$ is the only maximal element of $\sum$, which is not a minimal prime co-ideals of $I$.

\section{Total graph with respect to a co-ideal}

In this section we apply the results we have obtained about $S(I)$ in order to study the total graph with respect to co-ideal $I$. We begin with the key definition of this paper.

Definition. Let $R$ be a semiring and $I$ be a subtractive co-ideal of $R$. The total graph of $R$ with respect to co-ideal $I$ of $R$, denoted by $T\left(\Gamma_{I}(R)\right)$, is the graph with all elements of $R$ as vertices, and for distinct $x, y \in R$, the vertices $x$ and $y$ are adjacent if and only if $x y \in S(I) . S\left(\Gamma_{I}(R)\right)\left(\operatorname{resp} . \bar{S}\left(\Gamma_{I}(R)\right)\right)$ denotes the subgraph of $T\left(\Gamma_{I}(R)\right)$ with vertex set $S(I)$ (resp. $\left.\bar{S}(I)\right)$. 
Lemma 4.1. Let $R$ be a semiring and $I$ be a subtractive co-ideal of $R$. Then the following statements are equivalent:

(i) $T\left(\Gamma_{I}(R)\right)$ is an empty graph;

(ii) $S(I)=\{1\}$;

(iii) $I=\{1\}$ and $R$ is an I-semiring and a co-semidomain.

Proof. (i) $\Rightarrow$ (ii) Let $1 \neq x \in S(I)$. Then $x$ and 1 are adjacent in $T\left(\Gamma_{I}(R)\right)$, a contradiction.

(ii) $\Rightarrow$ (iii) Since $I \subseteq S(I), I=\{1\}$. Since $\{1\}$ is a co-ideal of $R, R$ is an $I$-semiring. Moreover, $\{1\}=S(I)=S(\{1\})=S(R)$. Hence $R$ is a cosemidomain.

(iii) $\Rightarrow$ (i) Let $x$ and $y$ be adjacent in $T\left(\Gamma_{I}(R)\right)$. So $x y \in S(R)=S(I)=\{1\}$. Hence $x=x(1+y)=x+x y=x+1=1$. By the similar way $y=1$. Thus $T\left(\Gamma_{I}(R)\right)$ is an empty graph.

A coclique in a graph $G$ is a set of pairwise nonadjacent vertices.

Proposition 4.2. Let $R$ be a semiring and $I$ be a subtractive co-ideal of $R$. Then

(i) Each $x \in \bar{S}\left(\Gamma_{I}(R)\right)$ and $y \in R$ are not adjacent;

(ii) $\bar{S}\left(\Gamma_{I}(R)\right)$ is a coclique in $T\left(\Gamma_{I}(R)\right)$;

(iii) $T\left(\Gamma_{I}(R)\right)$ is always disconnected.

Proof. The proof is similar to [13, Theorem 3.7].

As we see in Proposition 4.2, $T\left(\Gamma_{I}(R)\right)$ is always disconnected. In Theorem 4.4, we show that the subgraph $S\left(\Gamma_{I}(R)\right)$ of $T\left(\Gamma_{I}(R)\right)$ with vertex set $S(I)$ is connected. So we study only the subgraph $S\left(\Gamma_{I}(R)\right)$ of $T\left(\Gamma_{I}(R)\right)$.

Lemma 4.3. Let $R$ be a semiring and $I$ be a strong co-ideal of $R$. The following statements are equivalent:

(i) $S(I)$ is a strong co-ideal;

(ii) $S\left(\Gamma_{I}(R)\right)$ is a complete graph;

(iii) $\operatorname{diam}\left(S\left(\Gamma_{I}(R)\right)\right)=1$.

Proof. (i) $\Rightarrow$ (ii) and (ii) $\Rightarrow$ (iii) are clear.

(iii) $\Rightarrow$ (i) We show that $S(I)$ is a co-ideal of $R$. Let $x \in S(I)$ and $s \in R$. So $x+r \in I$ for some $r \in R \backslash I$. Since $I$ is a co-ideal of $R, x+s+r \in I$, which gives $x+s \in S(I)$. Now, let $x, y \in S(I)$. Since $\operatorname{diam}\left(S\left(\Gamma_{I}(R)\right)\right)=1, x y \in S(I)$, which implies $S(I)$ is a co-ideal of $R$.

Theorem 4.4. Let $R$ be a semiring and $I$ be a strong co-ideal of $R$. Then

(i) $S\left(\Gamma_{I}(R)\right)$ is a connected graph and $\operatorname{diam}\left(S\left(\Gamma_{I}(R)\right)\right) \in\{1,2\}$. Moreover, $\operatorname{diam}\left(S\left(\Gamma_{I}(R)\right)\right)=2$ if and only if $S(I)$ is not a co-ideal;

(ii) $\operatorname{gr}\left(S\left(\Gamma_{I}(R)\right)\right) \in\{3, \infty\}$.

Proof. The proof is similar to [13, Theorems 3.3 and 3.4]. For the moreover statement in (i) use Lemma 4.3. 
Theorem 4.5. Let $R$ be a semiring and $I$ be a subtractive co-ideal of $R$ such that $I \neq S(I)$. Then

(i) $|S(I)|=3$ if and only if $\operatorname{gr}\left(S\left(\Gamma_{I}(R)\right)\right)=\infty$;

(ii) $|S(I)| \geq 4$ if and only if $\operatorname{gr}\left(S\left(\Gamma_{I}(R)\right)\right)=3$.

Proof. (i) Let $\operatorname{gr}\left(S\left(\Gamma_{I}(R)\right)\right)=\infty$. By Lemma 3.1, $|S(I)| \neq 1$, 2. Suppose, on the contrary, $|S(I)| \geq 4$. Since the induced subgraph of $S\left(\Gamma_{I}(R)\right)$ with vertex set $I$ is a complete subgraph of $S\left(\Gamma_{I}(R)\right)$ and $\operatorname{gr}\left(S\left(\Gamma_{I}(R)\right)\right)=\infty$, we get $|I|=1$ or 2. We consider two cases:

Case 1: Let $|I|=1$. Since $|S(I)| \geq 4$ and $S(I)=I \cup S_{I}(R)$, we have $\left|S_{I}(R)\right| \geq 3$. So $\operatorname{diam}\left(\Gamma_{I}(R)\right)=2$ or 3 by Proposition 2.2. Hence there exist $x, y \in R \backslash I$ such that $d(x, y)=2$. So there exists $z \in R \backslash I$ such that $x-z-y$ is a path in $\Gamma_{I}(R)$. Thus $x, y \in(I: z)$, which gives $x, y, x y \in(I: z) \subseteq S(I)$, because $(I: z)$ is a co-ideal of $R$ by Proposition 2.1(ii). So $1-x-y-1$ is a cycle in $S\left(\Gamma_{I}(R)\right)$ and $\operatorname{gr}\left(S\left(\Gamma_{I}(R)\right)\right)=3$, a contradiction.

Case 2: Let $|I|=2$. Then $\left|S_{I}(R)\right| \geq 2$. If $\left|S_{I}(R)\right| \geq 3$, then by the similar argument as in case $1, \operatorname{gr}\left(S\left(\Gamma_{I}(R)\right)\right)=3$ a contradiction. Hence we assume $\left|S_{I}(R)\right|=2$. Let $1 \neq a \in I$ and $b, c \in S_{I}(R)$. Since $b$ and $c$ are the only elements of $S_{I}(R), b+c \in I$. So $a, b \in(I: c)$, which gives $a b \in(I: c) \subseteq S(I)$. So $1-a-b-1$ is a path in $S\left(\Gamma_{I}(R)\right)$, which implies $\operatorname{gr}\left(S\left(\Gamma_{I}(R)\right)\right)=3$, a contradiction.

Therefore $|S(I)|=3$.

Conversely, let $S(I)=\{1, a, b\}$. Since $S(I)$ is not a co-ideal of $R$ by Lemma $3.1, a b \notin S(I)$. Thus $a-1-b$ is the only path in $S\left(\Gamma_{I}(R)\right)$. So $\operatorname{gr}\left(S\left(\Gamma_{I}(R)\right)\right)=$ $\infty$.

(ii) It is clear by (i) and Theorem 4.4 and Lemma 3.1 .

The following example shows that the condition " $I$ is subtractive" in Theorem 4.5 is not superfluous.

Example 4.6. Let $R=(\{0,1,2,3\},+, \times)$, where

$$
a+b= \begin{cases}3 & \text { if } a, b \neq 0 \\ b & \text { if } a=0 \\ a & \text { if } b=0\end{cases}
$$

and $1 \times 1=1,2 \times 1=1 \times 2=2,3 \times 1=1 \times 3=3,2 \times 2=1,2 \times 3=3 \times 2=3$, $3 \times 3=3$, moreover $r \times 0=0 \times r=0$ for all $r \in R$. It can be easily seen that $I=\{1,3\}$ is a co-ideal of $R$, which is not subtractive. As we see, $S(I)=\{1,2,3\}$ and $\operatorname{gr}\left(S\left(\Gamma_{I}(R)\right)\right)=3$.

Proposition 4.7. Let $R$ be a semiring and $I$ be a subtractive co-ideal of $R$. If $a \in I$, then $a$ is adjacent to every vertex of $S\left(\Gamma_{I}(R)\right)$. Moreover, the converse is true if $\min (I)$ is finite.

Proof. Let $a \in I$ and $r \in S(I)$. By Theorem 3.7, $r \in P_{i}$ for some $P_{i} \in$ $\min (I)$. Hence $\operatorname{ar} \in P_{i} \subseteq S(I)$. Thus $a$ is adjacent to every vertex of $S\left(\Gamma_{I}(R)\right)$. Conversely, let $\min (I)$ be finite and $a$ be adjacent to every vertex of $S\left(\Gamma_{I}(R)\right)$. 
To prove our claim, we show $a \in P_{i}$ for each $P_{i} \in \min (I)$. By Lemma 3.9, for each $P_{i} \in \min (I)$, there exists $x_{i} \in P_{i}$ such that $x_{i} \notin \cup_{i \neq j} P_{j}$. As $a$ is adjacent to every other vertex and $x_{i} \in S(I), a x_{i} \in S(I)$. By Lemma 3.5, each $P_{i} \in \min (I)$ is subtractive. So $a x_{i} \notin P_{j}$ for each $P_{i} \neq P_{j} \in \min (I)$ by Proposition 2.1(ii). So $a x_{i} \in P_{i}$, which implies $a \in P_{i}$ by Proposition 2.1(ii). So $a \in P_{i}$ for each $P_{i} \in \min (I)$, which implies $a \in I$, by Proposition 2.1(iii).

The following example shows that the condition " $\min (I)$ is finite" in Proposition 4.7 is not superfluous.

Example 4.8. Let $R=\left(\mathbb{Z}^{+}, \operatorname{gcd}, \mathrm{lcm}\right)$ and $I=\{1\}($ take $\operatorname{gcd}(0,0)=0$ and $\operatorname{lcm}(0,0)=0)$. In Example 3.12, it is shown that $\min (I)$ is infinite. It can be easily seen that 2 is adjacent to every other vertex in $S\left(\Gamma_{I}(R)\right)$ and $2 \notin I$.

A vertex $x$ of a connected graph $G$ is a cut-point of $G$ if there are vertices $y$ and $z$ of $G$ such that $x$ is in every path from $y$ to $z$ (and $x \neq y, x \neq z$ ). Equivalently, for a connected graph $G, x$ is a cut-point of $G$ if $G-\{x\}$ is not connected.

The connectivity of a graph $G$, denoted by $\kappa(G)$, is defined to be the minimum number of vertices that are necessary to remove from $G$ in order to produce a disconnected graph.

Theorem 4.9. Let $R$ be a semiring and $I$ be a subtractive co-ideal of $R$. Then:

(i) $S\left(\Gamma_{I}(R)\right)$ has cut point if and only if $|\min (I)|=2$ and $I=\{1\}$.

(ii) If $V$ is the set of minimum number of vertices that are necessary to remove from $S\left(\Gamma_{I}(R)\right)$ in order to produce a disconnected graph, then $V \subseteq \cup V_{i}$, where $V_{i}=P_{i} \cap\left(\cup_{j \neq i} P_{j}\right)$ and $P_{i}, P_{j}^{\prime}$ s are minimal prime co-ideal of $I$. Moreover, $\kappa\left(S\left(\Gamma_{I}(R)\right)\right) \leq \min \left\{\left|V_{i}\right|\right\}$.

Proof. (i) Let $x$ be a cut-point of $S\left(\Gamma_{I}(R)\right)$. Thus $S\left(\Gamma_{I}(R)\right) \backslash\{x\}$ is not connected. Hence $I=\{x\}=\{1\}$, because if $I \backslash\{x\} \neq \emptyset$, then $S\left(\Gamma_{I}(R)\right) \backslash\{x\}$ is connected by Proposition 4.7, which is a contradiction. So $I=\{x\}=\{1\}$. Since $\{1\}$ is a co-ideal of $R, R$ is an $I$-semiring. Because $S\left(\Gamma_{I}(R)\right) \backslash\{1\}=$ $S(\Gamma(R)) \backslash\{1\}=S^{*}(\Gamma(R))$ is not connected, $|\min (R)|=2$ by Proposition 2.2 (ii).

Conversely, let $I=\{1\}$ and $|\min (R)|=2$. Then $S\left(\Gamma_{I}(R)\right)=S(\Gamma(R))$. It is clear that if we remove 1 from the vertex set of $S\left(\Gamma_{I}(R)\right)$, we gain the $S^{*}(\Gamma(R))$, which is disconnected by Proposition 2.2(ii). So $S\left(\Gamma_{I}(R)\right)$ has cut point 1 .

(ii) Let $V$ be the set of minimum number of vertices that are necessary to remove from $S\left(\Gamma_{I}(R)\right)$ in order to produce a disconnected graph and $V_{i}=P_{i} \cap$ $\left(\cup_{j \neq i} P_{j}\right)$. We show that $V \subseteq \cup V_{i}$. Suppose, on the contrary, there exists $x \in V$ such that $x \notin \cup V_{i}$. Let $V^{\prime}=S(I) \backslash V$. By definition of $V$, the induced subgraph with vertex set $V^{\prime}$ is not connected but the induced subgraph with vertex set $V^{\prime} \cup\{x\}$ is connected. Let $a, b \in V^{\prime}$ such that, there is no path between them. Since the induced subgraph with vertex set $V^{\prime} \cup\{x\}$ is connected, we have the path $a-x_{1}-x_{2}-\cdots-x_{n}-x-y_{1}-\cdots-y_{m}-b$. So $x x_{n}, x y_{1} \in S(I)$. 
Since $x \notin \cup V_{i}$, there are no minimal prime co-ideals $P_{i}, P_{j} \in \min (I)$ such that $x \in P_{i} \cap P_{j}$ (if $x \in P_{i} \cap P_{j}$, then $x \in V_{i}, V_{j}$, a contradiction). Hence there is only one minimal prime co-ideal $P_{i}$ such that $x \in P_{i}$. So $P_{i}$ is the only minimal prime co-ideal of $I$ such that $x x_{n}, x y_{1} \in P_{i}$ by Proposition 2.1(ii). This implies $x_{n}, y_{1} \in P_{i}$. So we have the path $a-x_{1}-\cdots-x_{n}-y_{1}-\cdots-y_{m}-b$ in the induced subgraph with vertex set $V^{\prime}$, which is a contradiction. Thus $V \subseteq \cup V_{i}$.

For the moreover statement we consider two cases:

Case 1: $\min (I)=\left\{P_{1}, P_{2}\right\}$, then $P_{1} \cap P_{2}=I$ by Proposition 2.1(iii). Since every element of $I$ is adjacent to each element of $P_{1}$ and $P_{2}$ by Proposition 4.7, we must remove all elements of $I$ to gain a disconnected graph. So $\kappa\left(S\left(\Gamma_{I}(R)\right)\right) \geq\left|P_{1} \cap P_{2}\right|=|I|$. Moreover, it can be easily seen that no elements of $P_{1} \backslash I=P_{1} \backslash P_{2}$ and $P_{2} \backslash I=P_{2} \backslash P_{1}$ are adjacent. So $\kappa\left(S\left(\Gamma_{I}(R)\right)\right)=\left|P_{1} \cap P_{2}\right|=|I|$.

Case 2: $|\min (I)| \geq 3$. Let $\min (I)=\left\{P_{i}\right\}_{i \in K}$ and $V_{i}=P_{i} \cap\left(\cup_{j \neq i, j \in K} P_{j}\right)$ for each $i \in K$. If for each $i \in K, V_{i}$ is an infinite set, then there is nothing to prove. Assume that there is an $i \in K$ such that $V_{i}$ is a finite set. Let $\left|V_{i}\right|=n$. We show that the induced subgraph with vertex set $S(I) \backslash V_{i}=V_{i}^{\prime}$ is not connected. It can be easily seen that $P_{i} \cap V_{i}^{\prime}=P_{i} \backslash \cup_{i \neq j} P_{j}$ and $P_{j} \cap V_{i}^{\prime}=P_{j} \backslash P_{i}$. We divide the proof into two steps:

Step 1: We claim that $V_{i}^{\prime} \cap P_{i} \neq \emptyset$. Because if $V_{i}^{\prime} \cap P_{i}=\emptyset$, then $V_{i}=P_{i}$. So $P_{i} \subseteq \cup_{i \neq j} P_{j}$. Since $\left|V_{i}\right|=n$ and $V_{i}=P_{i}, P_{i}=\left\{x_{1}, x_{2}, \ldots, x_{n}\right\}$. So there exists $P_{i} \neq P_{j}^{\prime} \in \min (I)$ such that $x_{j} \in P_{j}^{\prime}$ for each $x_{j} \in P_{i}$. So $P_{i} \subseteq \cup_{j=1}^{n} P_{j}^{\prime}$, which implies $P_{i} \subseteq P_{j}^{\prime}$ for some $1 \leq j \leq n$ by Lemma 3.9, a contradiction. Thus $V_{i}^{\prime} \cap P_{i} \neq \emptyset$ and $V_{i} \neq P_{i}$. Also, Since $P_{j}$ is a minimal prime co-ideal of $I$, $P_{j} \cap V_{i}^{\prime}=P_{j} \backslash P_{i} \neq \emptyset$.

Step 2: Let $a \in P_{i} \cap V_{i}^{\prime}$ and $b \in P_{j} \cap V_{i}^{\prime}$ (Note that $P_{i}$ is the only minimal prime co-ideal of $I$ with $a \in P_{i}$, because $\left.P_{i} \cap V_{i}^{\prime}=P_{i} \backslash \cup_{i \neq j} P_{j}\right)$. Now, we claim that there is no path between $a$ and $b$. Suppose, on the contrary, there is a path between $a$ and $b$. By Theorem 4.4, $a$ and $b$ are adjacent or $d(a, b)=2$. If $a$ and $b$ are adjacent, then $a b \in S(I)=\cup_{P_{j} \in \min (I)} P_{j}$. Since $P_{i}$ is the only minimal prime co-ideal of $I$ such that $a \in P_{i}, a b \in P_{i}$ by Proposition 2.1(ii). Hence $b \in P_{i}$, a contradiction. If $d(a, b)=2$, then there exists $c \in V_{i}^{\prime}$ such that $a-c-b$ is a path in $S\left(\Gamma_{I}(R)\right)$. Since $a c \in S(I)=\cup_{P_{j} \in \min (I)} P_{j}$ and $P_{i}$ is the only minimal prime co-ideal containing $a, a c \in P_{i}$. So $c \in P_{i} \cap V_{i}^{\prime}$. By the similar argument $P_{i}$ is the only minimal prime co-ideal containing $c$, which implies $b \in P_{i}$, a contradiction.

So there is no path between $a$ and $b$, which implies the induced subgraph with vertex set $V_{i}^{\prime}$ is not connected. Hence $\kappa\left(S\left(\Gamma_{I}(R)\right)\right) \leq \min \left\{\left|V_{i}\right|\right\}$.

Example 4.10. Let $X=\{a, b, c\}$ and $R=(P(X), \cup, \cap)$ a semiring with $1_{R}=X$, where $P(X)$ is the set of all subsets of $R$. If $I=\{X\}$, then $\min (I)=$ $\left\{P_{1}, P_{2}, P_{3}\right\}$, where $P_{1}=\{\{a\},\{a, c\},\{a, b\}, X\}, P_{2}=\{\{b\},\{b, c\},\{b, a\}, X\}$ and $P_{3}=\{\{c\},\{a, c\},\{b, c\}, X\}$. It can be easily seen that $\left|P_{i} \cap\left(P_{j} \cup P_{k}\right)\right|=3$ and $\kappa\left(S\left(\Gamma_{I}(R)\right)\right)=3$. 
Theorem 4.11. Let $R$ be a semiring and $I$ be a subtractive co-ideal of $R$. Then $S\left(\Gamma_{I}(R)\right)$ contains $|\min (I)|$ disjoint complete subgraphs.

Proof. Let $\min (I)=\left\{P_{i}\right\}_{i \in J}$. Set $V_{i}=P_{i} \backslash \cup_{i \neq j \in J} P_{j}$. Then $V_{i} \subseteq S(I)$ and $V_{i} \cap V_{j}=\emptyset$ for each $i \neq j$. Assume $G_{i}$ to be a subgraph of $S\left(\Gamma_{I}(R)\right)$ with vertex set $V_{i}$. It is clear that $G_{i}$ is a complete subgraph of $S\left(\Gamma_{I}(R)\right)$, because $P_{i} \in \min (I)$ is a co-ideal of $R$ for each $i \in J$. We show that $x$ and $y$ are not adjacent for each $x \in G_{i}, y \in G_{k}$. If $x$ and $y$ are adjacent, then $x y \in S(I)$. So $x y \in P_{t}$ for some $P_{t} \in \min (I)$. Thus $x, y \in P_{t}$ by Proposition 2.1(ii). Since $P_{i}$ is the only co-ideal containing $x$ and $P_{j}$ is the only co-ideal containing $y$, $P_{i}=P_{j}=P_{t}$. Thus $G_{i}=G_{j}$, which implies $V_{i}=V_{j}$, a contradiction.

Two graphs $G$ and $G^{\prime}$ with vertex set $V$ and $W$ respectively, are isomorphic if there is a bijection function $f: V \rightarrow W$ such that for all $v, w \in V(G)$ : $\{v, w\} \in E(G) \Leftrightarrow(f(v), f(w)) \in E\left(G^{\prime}\right)$.

Theorem 4.12. Let $R$ and $T$ be semirings and $I, J$ be co-ideals of $R$ and $T$ respectively with $\min (I)$ and $\min (J)$ are finite. If $S\left(\Gamma_{I}(R)\right) \cong S\left(\Gamma_{J}(T)\right)$, then $\Gamma_{I}(R) \cong \Gamma_{J}(T)$.

Proof. Let $f$ be a bijection (one-to-one correspondence) from $S(I)$ to $S(J)$. Since $S\left(\Gamma_{I}(R)\right) \cong S\left(\Gamma_{J}(T)\right),|S(I)|=|S(J)|$. So $\left|S_{I}(R) \cup I\right|=\left|S_{J}(T) \cup J\right|$. We claim that $\left|S_{I}(R)\right|=\left|S_{J}(T)\right|$. For this we show that $|I|=|J|$. Since $S\left(\Gamma_{I}(R)\right)=S\left(\Gamma_{J}(T)\right)$, there is a one-to-one corresponding between the vertices of $S\left(\Gamma_{I}(R)\right)$ and $S\left(\Gamma_{J}(T)\right)$. Let $a \in I$. By Proposition 4.7, $a$ is adjacent to every vertex of $S\left(\Gamma_{I}(R)\right)$. We show that $f(a) \in J$. Let $t \in S(J)$. So there exists $r \in S(I)$ such that $f(r)=t$. Because $a$ and $r$ are adjacent in $S\left(\Gamma_{I}(R)\right)$, $f(a)$ and $f(r)$ are adjacent in $S\left(\Gamma_{J}(T)\right)$. So $f(a)$ is adjacent to each element of $S\left(\Gamma_{J}(T)\right)$. Thus $f(a) \in J$ by Proposition 4.7. Hence $|I| \leq|J|$. By the similar way $|J| \leq|I|$. So $|I|=|J|$, which implies $\left|S_{I}(R)\right|=\left|S_{J}(T)\right|$. Now, let $a$ and $b$ are adjacent in $\Gamma_{I}(R)$. So $a+b \in I$. We claim that $f(a)$ and $f(b)$ are adjacent in $\Gamma_{J}(T)$. For this we show that $f(a)+f(b) \in J$. It suffices to show that for each $t \in S(J)(f(a)+f(b)) t \in S(J)$ by Proposition 4.7. Let $t \in S(J)$ and $f(s)=t$ for some $s \in R$. Since $a+b \in I,(a+b) s \in S(I)$ by Proposition 4.7. So $a s+b s \in S(I)=\cup_{i=1}^{n} P_{i}$. Thus $a s+b s \in P_{i}$ for some $P_{i} \in \min (I)$. Since $P_{i}$ is a prime co-ideal of $R$, as $\in P_{i}$ or $b s \in P_{i}$. Let $a s \in P_{i} \subseteq S(I)$. Thus $a$ and $s$ are adjacent in $S\left(\Gamma_{I}(R)\right)$ which implies $f(a)$ and $f(s)$ are adjacent in $S\left(\Gamma_{J}(T)\right)$. Hence $f(a) f(s) \in S(J)$. So $f(a) f(s) \in Q_{j}$ for some $Q_{j} \in \min (J)$. Thus $f(a) f(s)+f(b) f(s) \in Q_{j} \subseteq S(J)$, which gives $(f(a)+f(b)) t=(f(a)+f(b)) f(s) \in S(J)$, as needed.

\section{Total graph with respect to a Q-strong co-ideal}

In this section we investigate interrelation between $S\left(\Gamma_{I}(R)\right)$ and $S(\Gamma(R / I))$.

Proposition 5.1. Let $I$ be a $Q$-strong co-ideal of a semiring $R$ and let $x, y \in R$ such that $x \in q_{1} I$ and $y \in q_{2} I$ for some $q_{1}, q_{2} \in Q$. Then 
(i) $x$ is adjacent to $y$ in $S\left(\Gamma_{I}(R)\right)$ if and only if $q_{1} I$ is adjacent to $q_{2} I$ in $S(\Gamma(R / I))$. Moreover, $x \in S(I)$ if and only if $q_{1} I \in S(R / I)$.

(ii) $q I \cap S(I) \neq \emptyset$ if and only if all distinct elements of $q I$ are adjacent in $S\left(\Gamma_{I}(R)\right)$.

(iii) If $q I \cap S(I) \neq \emptyset$, then $q I \subseteq S(I)$.

(iv) $S\left(\Gamma_{I}(R)\right)$ contains at least $|S(I) \cap Q|$ disjoint complete subgraphs.

Proof. (i) Let $x$ be adjacent to $y$ in $S\left(\Gamma_{I}(R)\right)$. So $x y \in S(I)$, which implies $x y+r \in I$ for some $r \in R \backslash I$. Since $I$ is a $Q$-co-ideal of $R$, there exists the unique element $q_{3} \in Q$ such that $r \in q_{3} I$. Because $r \in q_{3} I$ and $r \notin I, I \neq q_{3} I$. Let $q_{1} I \odot q_{2} I \oplus q_{3} I=q_{4} I$. So $x y+r \in q_{4} I \cap I$, which gives $q_{4} I=I$. Hence $q_{1} I \odot q_{2} I \oplus q_{3} I=I$ which implies $q_{1} I \odot q_{2} I \in S(R / I)$. So $q_{1} I$ is adjacent to $q_{2} I$ in $S(\Gamma(R / I))$.

Conversely, let $q_{1} I$ be adjacent to $q_{2} I$ in $S(\Gamma(R / I))$. Hence $q_{1} I \odot q_{2} I \oplus q_{3} I=I$ for some $I \neq q_{3} I \in R / I$. This implies $x y+r \in I$ for each $x \in q_{1} I, y \in q_{2} I$ and $r \in q_{3} I$. So $x y \in(I: r) \subseteq S(I)$. Hence $x$ and $y$ are adjacent in $S\left(\Gamma_{I}(R)\right)$. The moreover statement is clear by similar argument.

(ii) Let $q I \cap S(I) \neq \emptyset$ and $x \in q I \cap S(I)$. Since $x \in S(I)$, there exists $r \in R \backslash I$ such that $x+r \in I$. Since $I$ is a $Q$-strong co-ideal of $R$, there exists the unique element $q^{\prime} \in Q$ such that $r \in q^{\prime} I$. Since $x+r \in\left(q I \oplus q^{\prime} I\right) \cap I$, $q I \oplus q^{\prime} I=I$. Thus $q I \in\left(\{I\}: q^{\prime} I\right)$. By Proposition 2.1(ii), $\left(\{I\}: q^{\prime} I\right)$ is a co-ideal which gives $q I \odot q I \in\left(\{I\}: q^{\prime} I\right)$. Thus $q I \odot q I \oplus q^{\prime} I=I$. Hence for each $a, b \in q I$ there exists $r \in q^{\prime} I$, such that $a b+r \in I$. Thus $a$ and $b$ are adjacent in $S\left(\Gamma_{I}(R)\right)$ for each $a, b \in q I$.

Conversely, let $x$ and $y$ be two elements of $q I$. By assumption $x$ and $y$ are adjacent in $S\left(\Gamma_{I}(R)\right)$. Hence $x y \in S(I)$, which gives $x, y \in S(I)$ by Lemma 3.1 (ii).

(iii) Let $x \in q I$ and $y \in q I \cap S(I)$. By (ii), $x$ and $y$ are adjacent. So $x y \in S(I)$, which gives $x \in S(I)$ by Lemma 3.1(ii). So $q I \subseteq S(I)$.

(iv) For each $q \in Q$, if $q \in S(I)$, then the induced subgraph of $S\left(\Gamma_{I}(R)\right)$ with vertex set $\{q I\}=\{q a: a \in I\}$ is a complete subgraph of $S\left(\Gamma_{I}(R)\right)$ by (ii). So $S\left(\Gamma_{I}(R)\right)$ contains at least $|S(I) \cap Q|$ complete subgraphs.

Theorem 5.2. Let $R$ be a semiring and $I$ be a $Q$-strong co-ideal of $R$.

(i) If $q I \in S(R / I)$, then $q I \subseteq S(I)$;

(ii) $S\left(\Gamma_{I}(R)\right)$ is complete if and only if $S(\Gamma(R / I))$ is complete;

(iii) $\operatorname{diam}\left(S\left(\Gamma_{I}(R)\right)\right)=1$ if and only if $\operatorname{diam}(S(\Gamma(R / I)))=1$;

(iv) $\operatorname{diam}\left(S\left(\Gamma_{I}(R)\right)\right)=2$ if and only if $\operatorname{diam}(S(\Gamma(R / I)))=2$;

(v) $\operatorname{gr}\left(S\left(\Gamma_{I}(R)\right)\right) \leq \operatorname{gr}(S(\Gamma(R / I)))$. Moreover, if $|\min (I)| \geq 3$, then

$$
\operatorname{gr}\left(S\left(\Gamma_{I}(R)\right)\right)=\operatorname{gr}(S(\Gamma(R / I))) .
$$

Proof. (i) If $S(R / I)=\{I\}$, then $R / I$ is a co-semidomain. Hence $I$ is prime by [10, Theorem 3.8]. This gives $I=S(I)$. Suppose that $S(R / I) \neq\{I\}$. Let $a \in I, q a \in q I$ and $q I \in S(R / I)$. Hence there exists $I \neq q^{\prime} I \in S(R / I)$ such that $q I \oplus q^{\prime} I=I$. Thus $q a+q^{\prime} b \in I$ for some $b \in I$, which implies $q a \in S(I)$. 
(ii) Let $S\left(\Gamma_{I}(R)\right)$ be complete and $q_{1} I, q_{2} I \in S(R / I)$. By (i), $q_{1} I, q_{2} I \subseteq$ $S(I)$, where $q_{i} I=\left\{q_{i} a: a \in I\right\}$. Hence $q_{1}, q_{2} \in S(I)$, which gives $q_{1} q_{2} \in S(I)$, because $S\left(\Gamma_{I}(R)\right)$ is a complete graph. So there exists $r \in R \backslash I$, such that $q_{1} q_{2}+r \in I$. Since $I$ is a $Q$-strong co-ideal of $R$, there exists a unique element $q_{3} \in Q$ such that $r \in q_{3} I$ (hence $q_{3} I \neq I$ ). If $q_{1} I \odot q_{2} I \oplus q_{3} I=q I$, then $q_{1} q_{2}+r \in q I \cap I$, which gives $q I=I$. This implies that $q_{1} I \odot q_{2} I \in(\{I\}$ : $\left.q_{3} I\right) \subseteq S(R / I)$. Thus $S(\Gamma(R / I))$ is a complete graph.

Conversely, let $S(\Gamma(R / I))$ be a complete graph. Let $x, y \in S(I)$. Since $I$ is a $Q$-strong co-ideal, there exist $q_{1}, q_{2} \in Q$ such that $x \in q_{1} I$ and $y \in q_{2} I$. Since $S(\Gamma(R / I))$ is a complete graph, $q_{1} I \odot q_{2} I \in S(R / I)$. Let $q_{1} I \odot q_{2} I=q_{3} I$, where $q_{3}$ is the unique element of $Q$ such that $\left(q_{1} q_{2}\right) I \subseteq q_{3} I$. So $x y \in q_{3} I$. Since $q_{3} I \subseteq S(I)$ (by (i)), $x y \in q_{3} I \subseteq S(I)$. Thus $S(I)$ is a complete graph.

(iii) and (iv) are clear by (ii) and Theorem 4.4.

(v) If $\operatorname{gr}(S(\Gamma(R / I)))=\infty$, there is nothing to prove. Let $\operatorname{gr}(S(\Gamma(R / I)))$ be finite. So $\operatorname{gr}(S(\Gamma(R / I)))=3$ by Theorem 4.4. Let $q_{1} I-q_{2} I-q_{3} I-q_{1} I$ be a path in $S(\Gamma(R / I))$ for some $q_{1} I, q_{2} I, q_{3} I \in S(R / I)$. By (i), $q_{1}-q_{2}-q_{3}-q_{1}$ is a path in $S\left(\Gamma_{I}(R)\right)$. So $\operatorname{gr}\left(S\left(\Gamma_{I}(R)\right)\right)=3$. Thus $\operatorname{gr}\left(S\left(\Gamma_{I}(R)\right)\right) \leq \operatorname{gr}(S(\Gamma(R / I)))$. For the moreover statement, let $|\min (I)| \geq 3$ and $P_{1}, P_{2}$ and $P_{3} \in \min (I)$. By Lemma 3.9, $P_{1} \not \subset P_{2} \cup P_{3}, P_{2} \nsubseteq \subseteq P_{1} \cup P_{3}$ and $P_{3} \not \subseteq P_{2} \cup P_{1}$. So $\{1, a, b, c\} \subseteq S(I)$ for some $a \in P_{1} \backslash P_{2} \cup P_{3}, b \in P_{2} \backslash P_{1} \cup P_{3}$ and $c \in P_{3} \backslash P_{2} \cup P_{1}$. So $\operatorname{gr}\left(S\left(\Gamma_{I}(R)\right)\right)=3$ by Theorem 4.5. We show that $\operatorname{gr}(S(\Gamma(R / I)))=3$. Since $I$ is a $Q$-strong co-ideal of $R$, there exist $q_{1}, q_{2}, q_{3} \in Q$ such that $a \in q_{1} I, b \in q_{2} I$ and $c \in q_{3} I$. Hence $a=q_{1} a_{1}$ and $b=q_{1} b_{1}$ for some $a_{1}, b_{1} \in I$. We show that $I \neq q_{1} I \neq q_{2} I \neq q_{3} I$. Let $q_{1} I=q_{2} I$. Since $a \in P_{1}$ and $P_{1}$ is subtractive, $q_{1} \in P_{1}$ by Proposition 2.1(ii). So $b=q_{1} b_{1} \in P_{1}$ because $q_{1}, b_{1} \in P_{1}$, a contradiction. Thus $I, q_{1} I, q_{2} I$ and $q_{3} I$ are distinct elements of $R / I$. By (i), $I, q_{1} I, q_{2} I$ and $q_{3} I \in S(R / I)$. So $|S(R / I)| \geq 4$, which implies $\operatorname{gr}(S(\Gamma(R / I)))=3$ by Theorem 4.4. Thus $\operatorname{gr}\left(S\left(\Gamma_{I}(R)\right)\right)=\operatorname{gr}(S(\Gamma(R / I)))$.

The following example shows that the condition " $|\min (I)| \geq 3$ " can not be omitted in Theorem 5.2.

Example 5.3. Let $X=\{a, b, c\}$ and $R=(P(X), \cup, \cap)$, where $P(X)=$ the set of all subsets of $X$. An inspection will show that $I=\{X,\{a, b\}\}$ is a $Q$-co-ideal of $R$, where $Q=\left\{q_{e}=X, q_{1}=\{b, c\}, q_{2}=\{a, c\}, q_{3}=\{c\}\right\}$ such that $q_{1} I=\{\{b\},\{b, c\}\}$ and $q_{2} I=\{\{a\},\{a, c\}\}$ and $q_{3} I=\{\{c\}, \emptyset\}$. It can be easily seen that $\min (I)=\left\{P_{1}, P_{2}\right\}$, where $P_{1}=\{\{a\},\{a, c\},\{a, b\}, X\}$ and $P_{2}=\{\{b\},\{b, c\},\{a, b\}, X\}$. We see that $S(R / I)=\left\{q_{e} I, q_{1} I, q_{2} I\right\}$ and $S(I)=P_{1} \cup P_{2}=\{X,\{a\},\{b\},\{a, c\},\{b, c\},\{a, b\}\}$. Hence $\{a\}-\{a, b\}-X-\{a\}$ is a cycle in $S\left(\Gamma_{I}(R)\right)$ and there is no cycle in $S(\Gamma(R / I))$. So $\operatorname{gr}\left(S\left(\Gamma_{I}(R)\right)\right)=3$ and $\operatorname{gr}(S(\Gamma(R / I)))=\infty$.

Theorem 5.4. Let $R$ be a semiring and I be a subtractive co-ideal of $R$, which is not prime. Then 
(i) $\omega\left(\Gamma_{I}(R)\right)<\omega\left(S\left(\Gamma_{I}(R)\right)\right)$ if and only if $\min (I)$ is finite with $3 \leq|\min (I)|$ or $|\min (I)|=2$ with $\left|P_{i}\right| \geq 3$, where $P_{i} \in \min (I)$.

(ii) $\omega\left(\Gamma_{I}(R)\right)=\omega\left(S\left(\Gamma_{I}(R)\right)\right)$ if and only if $|\min (I)|$ is infinite or $|\min (I)|=$ 2 with $\left|P_{i}\right|=2$ for each $P_{i} \in \min (I)$.

(iii) $\omega(S(\Gamma(R / I))) \leq \omega\left(S\left(\Gamma_{I}(R)\right)\right)$. Moreover, $\omega\left(S\left(\Gamma_{I}(R)\right)\right)=\omega(S(\Gamma(R / I)))$ if and only if $I=\{1\}$ or $\omega(S(\Gamma(R / I)))$ is infinite.

Proof. (i) Let $\omega\left(\Gamma_{I}(R)\right)<\omega\left(S\left(\Gamma_{I}(R)\right)\right)$. If $\omega\left(\Gamma_{I}(R)\right)$ is infinite, then $\omega\left(\Gamma_{I}(R)\right)$ $<\omega\left(S\left(\Gamma_{I}(R)\right)\right)$ implies that $\omega\left(S\left(\Gamma_{I}(R)\right)\right)$ is infinite. Hence

$$
\omega\left(\Gamma_{I}(R)\right)=\omega\left(S\left(\Gamma_{I}(R)\right)\right),
$$

a contradiction. Therefore $\omega\left(\Gamma_{I}(R)\right)$ is finite

By Proposition 2.2(iii), $\omega\left(\Gamma_{I}(R)\right)=|\min (I)|$. Hence $|\min (I)|$ is finite. We show that $3 \leq|\min (I)|$ or $|\min (I)|=2$ with $\left|P_{i}\right| \geq 3$, where $P_{i} \in \min (I)$. Since $I$ is not a prime co-ideal of $R,|\min (I)| \neq 1$ by Proposition 2.1(iii). Suppose on the contrary, $\omega\left(\Gamma_{I}(R)\right)=|\min (I)|=2$ and $\left|P_{1}\right|=\left|P_{2}\right|=2$. Let $P_{1}=\{1, a\}$ and $P_{2}=\{1, b\}$. By Theorem 3.7, $S(I)=\{1, a, b\}$ and $a b \notin S(I)$ by Lemma 3.1. Hence $a-1-b$ is the only path in $S\left(\Gamma_{I}(R)\right)$ and $\omega\left(S\left(\Gamma_{I}(R)\right)\right)=2$, a contradiction.

Conversely, if $\min (I)=\left\{P_{1}, P_{2}, \ldots, P_{n}\right\}$, where $n \geq 3$, then $\omega\left(\Gamma_{I}(R)\right)=n$ by Proposition 2.2(iii). By Proposition 2.1(iii), $I=P_{1} \cap P_{2} \cap \cdots \cap P_{n}$ and $I \neq \cap_{i=1, i \neq j}^{n} P_{i}$ for each $1 \leq j \leq n$. If $P_{1} \cap P_{j} \subseteq \cup_{i \neq 1, j} P_{i}$, then $P_{1} \cap P_{j} \subseteq P_{i}$ for some $1 \leq i \neq j \leq n$ by Lemma 3.9. This implies $P_{1} \subseteq P_{i}$ or $P_{j} \subseteq P_{i}$ by [10, Lemma 2.7], a contradiction. So $P_{1} \cap P_{j} \nsubseteq \cup_{i \neq 1, j} P_{i}$. Let $a_{j} \in P_{1} \cap P_{j} \backslash \cup_{i \neq 1, j} P_{i}$. Then $\left\{1, a_{1}, a_{2}, \ldots, a_{n}\right\} \subseteq P_{1}$, which gives $\left|P_{1}\right| \geq n+1$. Since the induced subgraph of $S\left(\Gamma_{I}(R)\right)$ with vertex set $P_{1}$ is a complete subgraph of $S\left(\Gamma_{I}(R)\right)$, $\omega\left(S\left(\Gamma_{I}(R)\right)\right) \geq n+1$. So $\omega\left(\Gamma_{I}(R)\right)<\omega\left(S\left(\Gamma_{I}(R)\right)\right)$. If $\min (I)=\left\{P_{1}, P_{2}\right\}$ with $\left|P_{i}\right| \geq 3$, then $\omega\left(\Gamma_{I}(R)\right)=2$ by Proposition $2.2($ iii). Since the induced subgraph with vertex set $P_{i}$ of $S\left(\Gamma_{I}(R)\right)$ is a complete subgraph of $S\left(\Gamma_{I}(R)\right)$ and $\left|P_{i}\right| \geq 3, \omega\left(S\left(\Gamma_{I}(R)\right)\right) \geq 3$. So $\omega\left(\Gamma_{I}(R)\right)<\omega\left(S\left(\Gamma_{I}(R)\right)\right)$.

(ii) Let $\omega\left(\Gamma_{I}(R)\right)=\omega\left(S\left(\Gamma_{I}(R)\right)\right)$. So $|\min (I)|$ is infinite or $|\min (I)|=2$ with $\left|P_{i}\right|=2$ for each $P_{i} \in \min (I)$ by (i).

Conversely, if $|\min (I)|$ is infinite, then $\omega\left(\Gamma_{I}(R)\right)=\infty$ by Proposition 2.2(iii). We show that every clique in $\Gamma_{I}(R)$ is a clique in $S\left(\Gamma_{I}(R)\right)$. Let $T$ be a clique in $\Gamma_{I}(R)$ and $x, y \in T$. It is clear that $x, y \in S(I)$ because $S_{I}(R) \subseteq S(I)$. Since $\omega\left(\Gamma_{I}(R)\right) \geq 3$, there exists $z \in T$ such that $x, y \neq z$. Since $T$ is a clique, $x, y \in(I: z)$ and so $x y \in(I: z) \subseteq S(I)$. So $T$ is a clique in $S\left(\Gamma_{I}(R)\right)$. Hence $\omega\left(S\left(\Gamma_{I}(R)\right)\right)=\infty$. If $|\min (I)|=2$ with $\left|P_{i}\right|=2$ for each $P_{i} \in \min (I)$, then $\omega\left(\Gamma_{I}(R)\right)=2$ by Proposition $2.2\left(\right.$ iii) and $\omega\left(S\left(\Gamma_{I}(R)\right)\right)=2$ by the proof of (i).

(iii) Let $\left\{q_{e} I, q_{1} I, \ldots, q_{n} I, \ldots\right\}$ be a clique in $S(\Gamma(R / I))$. Then $\left\{q_{e}, q_{1}, \ldots\right.$, $\left.q_{n}, \ldots\right\}$ is a clique in $S\left(\Gamma_{I}(R)\right)$ by Proposition 5.1(i). So $\omega(S(\Gamma(R / I))) \leq$ $\omega\left(S\left(\Gamma_{I}(R)\right)\right)$. For the moreover statement, it is clear that if $I=\{1\}$, then $\omega(S(\Gamma(R / I)))=\omega\left(S\left(\Gamma_{I}(R)\right)\right)$. If $\omega(S(\Gamma(R / I)))$ is infinite, then $\omega(S(\Gamma(R / I)))$ $=\omega\left(S\left(\Gamma_{I}(R)\right)\right)$, because $\omega(S(\Gamma(R / I))) \leq \omega\left(S\left(\Gamma_{I}(R)\right)\right)$. 
Conversely, let $\omega\left(S\left(\Gamma_{I}(R)\right)\right)=\omega(S(\Gamma(R / I)))$. Let $\omega(S(\Gamma(R / I)))$ be finite we show that $I=\{1\}$. We know that $q_{e} \in I$. We claim that for each $a \in I, a=q_{e}$. Suppose, on the contrary, there exists $a \in I$ such that $a \neq q_{e}$. Since $I$ is not prime, $R / I$ is not co-semidomain by [10, Theorem 3.8]. Hence $|S(R / I)| \geq 2$ which implies $\omega(S(\Gamma(R / I))) \geq 2$. Let $\left\{q_{e} I, q_{1} I, q_{2} I, \ldots, q_{n} I\right\}$ be a clique in $\omega(S(\Gamma(R / I)))$. By Proposition 4.7 and 5.1(i), $\left\{q_{e}, q_{1}, q_{2}, \ldots, q_{n}, a\right\}$ is a clique in $\omega\left(S\left(\Gamma_{I}(R)\right)\right)$, a contradiction. So for each $a \in I, a=q_{e}$. So $1=q_{e}$ and $I=\{1\}$.

Acknowledgment. We would like to thank the referee for several useful comments on the first draft of the manuscript.

\section{References}

[1] A. Abbasi and S. Habibi, The total graph of a commutative ring with respect to proper ideals, J. Korean Math. Soc. 49 (2012), no. 1, 85-98.

[2] D. F. Anderson and A. Badawi, The total graph of a commutative ring, J. Algebra 320 (2008), no. 7, 2706-2719.

[3] D. F. Anderson and P. S. Livingston, The zero-divisor graph of a commutative rings, J. Algebra 217 (1999), no. 2, 434-447.

[4] M. F. Atiyah and I. G. Macdonald, Introduction to Commutative Algebra, Addison Wesley Publishing Company, 1969.

[5] I. Beck, Coloring of commutative rings, J. Algebra 116 (1988), no. 1, 208-226.

[6] A. Bondy and U. S. R. Murty, Graph Theory, Graduate Texts in Mathematics, 244. Springer, New York, 2008.

[7] D. Dolžan and P. Oblak, The zero-divisor graphs of rings and semirings, Internat. J. Algebra Comput. 22 (2012), no. 4, 1250033, 20 pp.

[8] S. Ebrahimi Atani, The zero-divisor graph with respect to ideals of a commutative semiring, Glas. Mat. Ser. III 43(63) (2008), no. 2, 309-320.

[9] - An ideal-based zero-divisor graph of a commutative semiring, Glas. Matematicki 44(64) (2009), 141-153.

[10] S. Ebrahimi Atani, S. Dolati Pish Hesari, and M. Khoramdel, Strong co-ideal theory in quotients of semirings, J. Adv. Res. Pure Math. 5 (2013), no. 3, 19-32.

[11] The Identity-Summand Graph of Commutative Semirings, J. Korean Math. Soc. 51 (2014), no. 1, 189-202.

[12] _ A co-ideal based identity-summand graph of a commutative semiring, submitted.

[13] - Total Graph of a Commutative semiring with respect to identity-summand elements, J. Korean Math. Soc. 51 (2014), no. 3, 593-607.

[14] S. Ebrahimi Atani and F. Esmaeili Khalil Saraei, The total graph of a commutative semiring, An. Ştiinţ. Univ. "Ovidius" Constanţa Ser. Mat. 21 (2013), no. 2, 21-33.

[15] J. S. Golan, Semirings and Their Applications, Kluwer Academic Publishers Dordrecht, 1999.

[16] J. Kist, Minimal prime ideals in commutative semigroups, Proc. London Math. Soc. (3) 13 (1963), 31-50.

[17] H. Wang, On rational series and rational language, Theoret. Comput. Sci. 205 (1998), no. $1-2,329-336$ 
Shahabaddin Ebrahimi Atani

Faculty of Mathematical Sciences

University of Guilan

P.O. Box 1914, RASHT, Iran

E-mail address: ebrahimi@guilan.ac.ir

Saboura Dolati Pish Hesari

Faculty of Mathematical Sciences

UNIVERSITY OF GUILAN

P.O. Box 1914, RASHT, IraN

E-mail address: saboura_dolati@yahoo.com

Mehdi Khoramdel

Faculty of Mathematical Sciences

University of GuILAN

P.O. Box 1914, Rasht, IraN

E-mail address: mehdikhoramdel@gmail.com 